\title{
Index autorum ad Vol. 151
}

Confecit F. Ammann, Geneve

(B) $=$ Buchbesprechungen $-(\mathrm{V})=$ Vortrag - C

- $\quad$ Livres nouveaux - Book reviews

- $\quad$ Communication - Report

Adelstein, F, 465

Allen, L., 615 (B)

Andrade, L. de, v. De Andrade, L.

Arkhangelsky, V. N., 260 (V)

Ashlon, N., 97 (V)

Babel, J., 405 (V)

Bacskulin, Elisabeth, 555

Bacskulin, J., 555

Barishak, B., 184 (V)

Bechrakis, E., 604

Bejach, H. E. (Editor), 535 (B)

Bertelsen, T. I., 309 (V)

Blodi, F. C, 615 (B)

Böck, J., 331 (V)

Brihaye-van Geertruvden, M., 627 (V)

Brown, E. E., 534 (B)

Bruckner, A., 663 (B)

Casanovas, J., 272 (V) Cerletti, A., 512 Charamis, J., 161 (V) Colenbrander, A., 646 (V)

Custodis, E., 623 (V)

Dake, C. L, 620 (V)

Darabos, G., 477

De Andrade, L., 427 (V)

De Jong, H., 627 (V)

Dekking, H. M., 534 (B)

Delleman, J. W., 625 (V)

Deucher, F., 535 (B)

Dijk, J. R. van, v. Van Dijk, J. R.

Dollfus, M. A., 23 (V)

Ehlers, H., 284 (V)

Fattorusso, V., 535 (B) Feyrter, F., 331 (V) Francois, J. (Editor), 1-456 (V) Francois, J., 114 (V), $135(\mathrm{~V})$

Gall, J., 1 (V)

Gramberg-Danielsen, B., 663 (B) Gunzert, 663 (B)

Hagedoorn, A., 618 (V), 634 (V) Hanssens, M., 135 (V) Hemmes, G. D., 197 (V) Hewson, G. E., 51 (V) Hockwin, 0., 505 Hönig, Maria, 580 Hollwich, F., 615 (B) 
Jaensch, P. A, 615 (B)

Jancar, J., 548

Jong, $\mathrm{H}$. de, v. De Jong, $\mathrm{H}$.

Kappert, A., 535 (B) Klika, E., 568 Klöti, R, 482 Kloucek, F., 568

Lemke, L., 457 Litriõin, O., 390 (V)

Meier-Ruge, W, 512 Moro, F., 349 (V) Müller, G., 535 (B) Müller-Jensen, 663 (B) Newsom, W.

A., 505 Niesel, P., 489 Nijland, R., 197 (V)

Päcurariu, I., 75 (V) Pilleri, G., 535 (B) Planten, J. Th., 655 (V)

Radnót, M., 1 (V) Rintelen, F, 58 (V) Ritter, O., 535 (B) Rohrschneider, W., 663 (B) Rossi, E.

(Editor), 536 (B) Rudler, J. C., 535 (B)

Sachsenweger, R., 662 (B) Sampimon, R. L. H., 637 (V) Schappert-Kimmíjser, J., 197 (V)

Schmachtenberg, 663 (B) Schreiber, G. (Editor) , 535 (B) Schwab, F., 231 (V)

672

Index autorum ad Vol. 151

Sezer, N., 184 (V) Shalsha, K. G. (Editor), 535 (B) Stallard, H. B., 214 (V) Stankovic, I., 390 (V)

Stefanovic, P., 390 (V) Streiff, E. B., 535-536 (B), 615 (B), 662-663 (B)

Thiel, B., 68 (V) Topalis, C, 161 (V)

Valle, O., 537

Van Dijk, J. R., 629 (V)

Velzeboer, C. M. J., 627 (V)

Vereecken, E., 465

V. Vos, J. J., 652 (V)

Wandeler, A., 535 (B) Winkelman, J. E., 625 (V) Woltei·, J. R., 588 Worst, J. G. F., 659 (V) 